\title{
The Incidence Of Out-Of-Body Experiences In Hospitalized Patients
}

\author{
Melodie Olson, R.N., Ph.D. \\ College of Nursing, Medical University of South Carolina
}

ABSTRACT: The author studied out-of-body experiences (OBEs), cultural differences in reporting those OBEs, and associations between OBEs and frequency of dream recall in 100 white and 100 black adults hospitalized in a university medical center. Of the 200 subjects interviewed, 31 reported having had an OBE, six during the current hospitalization. White patients reported OBEs significantly more often than did black patients, possibly because of methodological problems in the interview techniques. Chi-squared tests showed significant positive associations between frequency of dream recall and incidence of OBEs, and between vivid dreams and incidence of OBEs.

When a person feels his or her mind, consciousness, or center of awareness to be at a place different from the physical body, it is termed an out-of-body experience (OBE). It is different from a hallucination or schizophrenic loss of body boundaries in that the person seems well based on other mental health parameters. It is different from depersonalization in that it is not accompanied by extreme anxiety, but is calming and satisfying (Gabbard \& Twemlow 1984). And OBErs state the OBE is not a dream, but is much more realistic than even vivid dreams, and frequently occurs while awake (Green, 1968; Gabbard \& Twemlow 1984).

Current literature provides a great deal of case-study information about the out-of-body experience in relation to the near-death experience (NDE). The out-of-body experience occurs not only within the near-death experience but at other times as well. Persons who medi-

Dr. Olson is Associate Professor of Graduate Medical-Surgical Nursing at the Medical University of South Carolina. Requests for reprints should be addressed to Dr. Olson at the College of Nursing, Medical University of South Carolina, 171 Ashley Avenue, Charleston, SC 29425-2404. 
tate frequently, others who are in the hypnogogic state, some who use drugs, and many who are anesthetized report the experience (Muldoon \& Carrington, 1974). Susan Blackmore (1982) listed at least ten surveys identifying the incidence of $\mathrm{OBE}$ in students, townspeople (Charlottesville, VA), members of special groups and national groups. Incidence rates vary from eight percent to as much as $44 \%$ (marijuana users) and 50\% (members of the Association for Research and Enlightment). Laboratory studies and surveys have shown that during (and sometimes after) out-of-body experiences, a person may have physiologic as well as affective change (Tart, 1968; 1967). The near-death studies have documented that the out-of-body experience does occur in the hospital setting and health care personnel frequently care for patients who have had them (Sabom, 1982). For these reasons, the study of out-of-body experiences is of legitimate concern to those who care for patients in hospitals.

This study was designed primarily to begin to establish the incidence of the OBE in hospitalized patients. The definition of OBE used here was used in several other studies (e.g., Palmer, 1979), and reflects the OBE as the "feeling of being outside of the body." This definition is somewhat analogous to the popular definition of pain: "Pain is what the patient says it is" (Mettler, 1964). Both definitions rely on selfreport. Yet in the case of the $\mathrm{OBE}$, investigators suggest that there are no empirical or conceptual grounds to restrict the definition.

A second purpose of this study was to determine cultural difference in reporting OBEs between black and white patients in a university hospital. This purpose was identified because the sample included a unique group of people, described in the discussion section of this paper. $\mathrm{OBE}$ literature documents that the $\mathrm{OBE}$ occurs in most cultural groups studied.

Because the identification of persons who have had OBEs was inherent in this study, the opportunity to investigate at least one characteristic OBErs have in common was irresistible. So a third purpose was to determine whether persons who have OBEs also remember dreams frequently, or have vivid dreams. Gabbard \& Twenlow (1984) cited studies by a number of researchers that suggest a correlation between OBE and frequency of remembering dreams. Questions related to vividness of dreaming and frequency of remembering dreams were included in the interview to support previous studies in this area.

\section{Method}

Sample:

Alert, adult, medical or surgical patients were interviewed after informed consent was obtained. One hundred subjects were white, 100 
were black. Females accounted for $54.5 \%$ of the sample (109 subjects), males for $45.5 \%$ (91 subjects). No attempt at systematic randomization was made, and the use of a convenience sample was considered a limitation of the study.

\section{Procedure:}

The investigator approached adult patients on medical surgical units who were in their rooms or pointed out by staff as potential interviewees. The nature of the study was explained, including the fact that it concerned the $\mathrm{OBE}$. Then patients were asked to sign a consent form indicating their willingness to participate. The short interview followed, asking if the person ever had an OBE. If he or she reported having had an OBE, information about the frequency of remembering dreams and the vividness of dreams was requested. The subject was also asked to describe the OBE.

\section{Demographic data:}

Demographic information was collected from patients' charts relating to sex, age, race, diagnosis, and medications taken within 24 hours of the interview. Medications were recorded because certain medications taken before the interview could influence the ability of the patient to understand the questions.

\section{Results}

Thirty-one subjects (15.5\% of the sample) reported having had OBEs at some time in their lives. Another nine subjects (4.5\%) were unsure whether or not they had had OBEs. The remaining 160 subjects $(80 \%$ denied ever having had an OBE.

Of the 31 subjects reporting having had an OBE, ten were black and 21 were white. When those who were unsure were classified as nonOBErs, a significantly greater number of white subjects than black reported having had OBEs ( $p=.0507$ ), as assessed by chi-square.

The anecdotal reports of the OBEs described by respondents in the study were analyzed. Of the 31 persons who admitted having had an OBE, six reported OBEs during the present hospitalization (three percent of the total sample of patients), 11 reported OBEs during a previous hospitalization, 12 reported OBEs out of the hospital, and two could not specify whether their OBEs occurred in or out of the hospital.

Of those 31 OBEs subjects who described OBEs, 22 reported OBEs related to stress, six reported OBEs related to relaxation, and the 
remaining three could not specify whether their OBEs were related to stress or relaxation. Nine of the 31 OBErs (4.5\% of the total sample) reported OBEs occurring in a near-death situation, 16 reported OBEs not occurring in a near-death situation, and the remaining six could not specify whether or not their OBEs occurred while they were near death.

Persons who reported OBEs also stated that they remembered dreams more frequently than did non-OBErs $(p=.0084)$, and reported more vivid dreams than did non-OBErs $(p=.0044)$, as assessed by chisquare.

\section{Discussion}

The figure of $15.5 \%$ of the sample of 200 subjects reporting an OBE is within the reported percentages of OBEs in general population groups gathered by various surveys (14-32\%). The percentage of OBEs reported by the 100 white subjects $(21 \%)$ is also consistent with published survey data. However, the percentage of OBEs reported by the original sample of 100 black subjects (ten percent) is below the reported range.

The sample originally was divided into 100 blacks and 100 whites because the black population served by the clinical facility used for the study includes Gullah people. The Gullah are a group of black citizens who have retained cultural uniqueness, including dialect and health belief system from pre-Civil War days. It was thought that certain of their cultural beliefs might be confused with the OBE. It should be noted that not all the black population admitted to this hospital are Gullah, and no statistics were included here to determine the size of the Gullah influence on the black population. But their presence in the population cannot be disregarded. Cross-cultural studies of the OBE indicate the experience is common in all cultures studied, so it was felt that more blacks would answer the OBE question affirmatively than whites because of the additive effects of the normal incidence of OBEs in the black population and the special beliefs among cultural groups in this area. Initial results showed a difference between the races $(p=0.05)$ in the opposite direction.

Literature suggests that the race of an interviewer does not matter if the questions asked are of a factual nature. That is, interviewees are likely to give their correct age or address to an interviewer of a race similar to or different from their own. But if the question is emotional in nature, interviewees are more likely to respond accurately to an interviewer of their own race (Cotter, Cohen \& Coulter, 1982). They 
will tell the interviewer of another race what they think that person wants to hear. It is logical to assume that there is some emotional content to the $\mathrm{OBE}$, and to reporting it, as evidenced by case study data. This emotional content may influence the response of the interviewee to interviewers of a different race. For that reason, it is possible that the incidence of OBEs reported in the sample of 200 patients of $15.5 \%$ is low. In future studies of this kind, consideration should be given to the race of data collectors and the race of their interviewees.

Anecdotal information about the six persons who had an OBE during the current hospitalization show a continuum of experiences from completely undramatic to intensely emotional. One young woman (21 years old), admitted for a gunshot would to her chest and abdomen, remembered floating above her body in the emergency room watching hospital personnel cut off her garter belt. She remembered viewing the tops of peoples' heads with curiosity, and knew she felt calm. But she only remembered the "overhead shot" and the experience after being asked about it by the interviewer. It was so undramatic that she had not thought of it since it happened.

At the other end of the continuum was a young man ( 30 years old) admitted to the hospital after a traumatic accident, who had had a classic near-death experience. He reported that he was "floating above his body," went to a "bright place," saw visions of people he knew, especially his dead mother, and was told he could not stay. He awoke on the operating room table. He cried during much of the interview, and related that his whole life was changed. He had not told any hospital or medical staff of his experience. Because he was obviously so intensely emotional at the time of the interview, the interviewer requested and received his permission to tell the staff he had had an intense emotional experience so that they would be available for continued support. It should be noted that the interview took place about three weeks after his "experience," and his medical condition was such that he was expected to recover fully.

Of the other four patients relating OBEs during this hospitalization, two were related to emotional states occurring while lying in bed (e.g., one felt she "got lost, wandered away from my body, could not help myself and became very uncomfortable"). Of the six OBErs, four found the experience growth producing or at least curious and calming. Two became frightened during the experience.

Other results of this study that show similarities between dreamers and OBErs are not new (Blackmore, 1982). They support data from existing studies, however, and suggest that studying OBEs in relation to phenomena like dreams, or studying characteristics of dreamers and 
OBErs, might give useful data concerning the nature of the OBE, or the characteristics of people who have them.

\section{Conclusions}

The lifetime incidence of OBEs in this group of hospitalized patients was $15.5 \%$; the incidence of OBEs occurring while these patients were in the hospital was $8.5 \%$; and the incidence of OBEs occurring during this single, identified hospital stay was three percent.

Race seemed to be a significant factor in the reporting of OBEs in this study; consequently, I recommend that when an interview method is employed in studying OBEs, data collectors and subjects should be of the same race.

Finally, the significant correlations between OBEs and frequency of dream recall, and between OBEs and dream vividness, suggest further study of those relationships.

\section{References}

Cotter, P. R., Cohen, J., \& Coulter, P. B. (1982). Race-of-interviewer effects in telephone interviews. Public Opinion Quarterly, 46, 278-284.

Blackmore, S. J. (1982). Beyond the Body. London, England: Heinemann.

Gabbard, G. O., \& Twemlow, S. W. (1984). With the Eyes of the Mind. An Empirical Analysis of Out-of-Body States. New York, NY: Praeger.

Green, C. (1968). Lucid Dreams. London, England: Hamish Hamilton.

Mettler, F. A. (1964). Pain. I: What is it? Journal of the Medical Society of New Jersey, 61 , 10.

Muldoon, S. J., \& Carrington, H. (1974). The Projection of the Astral Body. New York, NY: Samuel Weiser.

Palmer, J. (1979). A community mail survey of psychic experiences. Journal of the American Society for Psychical Research, 73, 221-251.

Sabom, M. B. (1982). Recollections of Death: A Medical Investigation. New York, NY: Harper \& Row.

Tart, C. (1967). A second psychophysiological study of out-of-the-body experiences in a gifted subject. International Journal of Parapsychology, 9, 251-258.

Tart, C. (1968). A psychophysiological study of out-of-body experiences in a selected subject. Journal of the American Society for Psychical Research, 62, 3-27. 